\title{
Caracterização da interação genótipo-ambiente e comparação entre modelos para ajuste do ganho pós-demama de bovinos Devon via normas de reação ${ }^{1}$
}

\author{
Marcela Bicca Bragança Corrêa², Nelson José Laurino Dionello ${ }^{3}$, Fernando Flores Cardoso4 \\ 1 Pesquisa financiada pela Embrapa (projeto 03.04.3.26) e pelo CNPq (processo 481565/2004-3). \\ 2 Doutoranda do Departamento de Zootecnia/FAEM/UFPEL. \\ 3 Departamento de Zootecnia/FAEM/UFPEL. \\ ${ }^{4}$ Embrapa Pecuária Sul - Bajé, RS. Bolsista produtividade do CNPq.
}

RESUMO - Os objetivos com este estudo foram verificar a presença de interação genótipo-ambiente e comparar modelos para o ajuste do ganho pós-desmama padronizado para 345 dias de bovinos da raça Devon, via normas de reação obtidas por regressão aleatória. Foram utilizados 14.973 registros coletados pelo Programa de Melhoramento de Bovinos de Carne entre 1980 e 2005. Para análise dos dados, foi utilizado o programa INTERGEN. Primeiramente, foi rodado o modelo animal convencional (MA), para comparação e estimativas do gradiente ambiental, com base nos desvios dos grupos de contemporâneos, e, em seguida, rodados os modelos hierárquicos de normas de reação com variâncias residuais homogêneas (MHNR) e heterogêneas (MHNRH). Nesses dois últimos, foram incluídos efeitos fixos de idade da vaca e idade do bezerro ao sobreano e efeitos aleatórios de grupos de contemporâneos e genéticos (nível e inclinação da norma de reação). No MHNR, as estimativas da variância residual, genética aditiva do nível e da inclinação da norma de reação e sua correlação genética foram, respectivamente, $272,02 \pm 8,51 ; 340,38 \pm 17,11 ; 0,119 \pm 0,006$ e $0,83 \pm 0,01$ e, no MHNRH, foram 401,96 $\pm 11,91$; $177,86 \pm 16,60 ; 0,076 \pm 0,006$ e 0,72 $\pm 0,02$, respectivamente. A herdabilidade, bem como a variância genética, foi crescente no gradiente ambiental, caracterizando a existência de interação genótipo-ambiente. Com base nos Critérios de Informação Deviance, nos Fatores de Bayes e Pseudo Fatores de Bayes, o MHNR proporcionou melhor ajuste aos dados, sendo o mais apropriado para considerar a interação genótipo-ambiente dessa população.

\section{Palavras-chave: avaliação genética, bovinos de corte, heterogeneidade de variâncias, inferência bayesiana, regressão aleatória \\ Genotype by environment interaction characterization and model comparison for post weaning gain adjustment of Devon cattle via reaction norms}

\begin{abstract}
The objectives of this study were to verify the presence of the genotype by environment interaction and to compare different models to fit Devon cattle post weaning gain standardized to 345 days, via reaction norms obtained by random regression. Data from 14,973 calves collected by the beef cattle improvement program from 1980 to 2005 were used. The INTERGEN program was used for data analysis. First, a standard animal model (AM) was fitted to serve as comparison basis and to provide estimates of the environmental gradient, based on contemporary group (CG) deviations and, next, the reaction norms hierarchical models with homogeneous (RNHM) and heterogeneous (RNHMH) residual variances were run. In these last two models, fixed effects of age of dam and age of calf at yearling and random contemporary group and genetic effects (level and slope of animal's reaction norms) were included. For the RNHM estimates of residual variance, reaction norm level and slope additive genetic variances and their genetic correlation were, respectively, $272.02 \pm 8.51 ; 340.38 \pm 17.11 ; 0.119 \pm 0.006$ and $0.83 \pm 0.01$, while for the RNHHM were $401.96 \pm 11.91$; $177.86 \pm 16.60 ; 0.076 \pm 0.006$ and $0.72 \pm 0.02$, respectively. The heritability asa well as the genetic variance increased on the environmental gradient, characterizing that there is genotype by environment interaction. Based on the Deviance Information Criterion, Bayes Factors and Pseudo Bayes Factors, the NRHM provided superior fit to the data, being the most appropriate model to consider the genotype by environmental interaction of this population.
\end{abstract}

Key Words: Bayesian inference, beef cattle, genetic evaluation, heterogeneity of variance, random regression 


\section{Introdução}

A cadeia produtiva de bovinos de corte, cada vez mais organizada e especializada, tem tido crescente destaque no agronegócio brasileiro, levando o País a ser atualmente o maior exportador de carne bovina do mundo. Neste contexto, a seleção é uma ferramenta de melhoramento genético que permite obter maior produtividade e eficiência econômica pela escolha de reprodutores com mérito genético superior.

Contudo, as avaliações genéticas atualmente não contemplam a verificação de interação genótipo-ambiente ( $\left.\mathrm{G}^{*} \mathrm{~A}\right)$ e utilizam metodologias em que se pressupõem homogeneidade de variâncias. Entretanto, diversos pesquisadores (Boldman \& Freeman, 1990; Dong \& Mao, 1990; Reverter et al., 1997; Torres, 1998) relatam a existência da heterogeneidade de variâncias entre rebanhos ou grupo de contemporâneos (Cavalheiro et al., 2002). Essa heterogeneidade, quando ignorada, pode ocasionar redução da resposta à seleção (Garrick \& Van Vleck, 1987).

A interação $G^{*} A$, quando desconsiderada, também pode prejudicar o ganho genético por causar alterações nas estimativas de parâmetros genéticos e variâncias, resultando em equívocos na escolha dos reprodutores. Portanto, animais identificados como melhoradores de determinado rebanho podem não ser os melhores se transferidos para condições ambientais diferentes ou se sua progênie for criada em condição ambiental diferente daquela onde foram selecionados.

Os modelos de regressão aleatória (MRA) têm sido propostos como alternativa na avaliação genética de dados longitudinais por permitirem ajustar uma trajetória aleatória para cada indivíduo como desvios de uma trajetória média da população, possibilitando que cada animal tenha uma forma diferente da trajetória de seus desempenhos genéticos (Mercadante et al., 2002).

Por meio das funções de covariância é possível determinar a norma de reação dos animais e as variações gradativas no ambiente de produção, que podem ser obtidas pela regressão do desempenho dos genótipos no valor médio de desempenho observado em cada ambiente (De Jong, 1995; Falconer \& Mackay, 1996). Assim, é possível identificar os genótipos de melhor desempenho em cada extrato ambiental e generalizar esse desempenho para um número infinito de ambientes, além de remover o limite de número de registros e permitir considerar a heterogeneidade de variâncias (Meyer, 2003).

Considerando que o ambiente de criação nos principais países fornecedores de animais com avaliação do mérito genético de bovinos é bastante diferente das condições de pastejo extensivo predominantes no Sul do Brasil, objetivou-se com este estudo verificar a presença da interação $\mathrm{G}^{*} \mathrm{~A}$ em rebanhos da raça Devon no estado do Rio Grande do Sul, por meio de normas de reação obtidas por regressão aleatória, e avaliar qual modelo proposto melhor se ajusta aos dados.

\section{Material e Métodos}

O estudo foi realizado utilizando-se dados de campo de bovinos da raça Devon participantes do PROMEBO Programa de Melhoramento de Bovinos de Carne, conduzido pela ANC - Associação Nacional de Criadores "Herd Book Collares" - entre os anos de 1980 e 2005. Foram utilizados 25.500 registros e, desses registros, restaram 14.973 animais conectados (compartilham laços genéticos) para as análises.

A característica avaliada foi o ganho de peso pósdesmama padronizado para 345 dias de idade (GPD345). Inicialmente, foram realizadas análises de preparação, formatação e descrição dos dados, usando rotinas criadas por Cardoso (2008) em linguagem SAS (SAS, 2002). Poste-riormente, foi testada a conexão dos grupos de contemporâneos, com base no número total de laços genéticos (mínimo 10), usando o programa AMC (Roso \& Schenkel, 2006). Foi feita a leitura da saída do programa AMC e a preparação do arquivo de pedigree e o arquivo de dados, incluindo somente grupo de contemporâneos conectados, para iniciar a análise genética sob modelo animal. Também foram testadas a significância e pertinência do modelo fixo proposto antes de rodar o programa INTERGEN (Cardoso, 2008).

Os grupo de contemporâneos foram formados para agrupar os animais que tiveram ambiente comum ou equivalente para expressar seu potencial produtivo (rebanhoano-estação-código de manejo-data da pesagem e sexo).

A seguir, foi utilizado o programa INTERGEN com um modelo animal na obtenção das estimativas dos efeitos médios de ambiente (grupos de contemporâneos) e também para servir de base de comparação para a análise de normas de reação.

O programa INTERGEN ajusta modelos hierárquicos de Bayes, ou seja, com parâmetros definidos em níveis ou estágios estruturados, que contemplem a diversidade de situações comuns em dados de desempenho animal e, no caso dos modelos de normas de reação, o valor genético do animal é obtido por uma função do nível ambiental médio correspondente à solução do grupo de contemporâneos ao qual o registro pertence, isto é, para cada nível ambiental, há um valor genético específico de cada animal, seguindo a estrutura geral abaixo descrita, que acrescenta os efeitos 
aleatórios da norma de reação ao modelo animal anteriormente utilizado:

$$
y_{i j}=\mu+g c_{j}+\mathbf{x}_{i}^{\prime} \boldsymbol{\beta}+a_{i}+b_{i} \hat{X}_{(g c) j}+e_{i j},
$$

em que $y_{i j}=$ registro de produção do animal $i$ pertencente ao grupo de contemporâneos $j ; \mu=$ média geral; $g c_{j}=$ efeito do $\mathrm{GC} j ; \boldsymbol{\beta}=$ vetor de efeitos fixos (coeficientes para idade da vaca e do bezerro lineares e quadráticos); e $\mathbf{x}_{i}^{\prime}=$ vetor de incidência de efeitos fixos; $a_{i}$ e $b_{i}=$ respectivamente, o intercepto e coeficiente de regressão linear aleatórios correspondentes à norma de reação do animal $i$ ao ambiente representado por $\hat{X}_{(g c) j}$, que é determinado pela solução do efeito de grupo de contemporâneos, previamente obtida por meio de um modelo animal; e $e_{i j}=$ erro aleatório.

Os efeitos de grupo de contemporâneos foram reestimados considerando a presença de interação genótipoambiente, adotando-se a seguinte pressuposição: $g c_{j} \sim N\left(0, \sigma_{g c}^{2}\right)$, para todo $j$.

Os efeitos aleatórios correspondentes a $q$ animais, $\mathbf{a}=\left\{a_{i}\right\}_{i=1, q}$ e $\mathbf{b}=\left\{b_{i}\right\}_{i=1, q}$, são presumidos com distribuição normal de média nula e matriz de covariância dada por:

$$
V\left[\begin{array}{l}
\mathbf{a} \\
\mathbf{b}
\end{array}\right]=\left[\begin{array}{cc}
\sigma_{a}^{2} & \sigma_{a b} \\
\sigma_{a b} & \sigma_{b}^{2}
\end{array}\right] \otimes \mathbf{A},
$$

na qual: $\sigma_{a}^{2}$ e $\sigma_{b}^{2}=$ variâncias genéticas aditivas; $\sigma_{a b}=$ covariância genética entre intercepto e inclinação da norma de reação, e $\mathrm{A}=$ matriz do numerador de parentesco.

Também foi rodado no programa INTERGEN o mesmo modelo hierárquico de normas de reação, porém heterocedástico (MHNRH), para permitir, além de variância genética heterocedástica no gradiente ambiental já contempladas no MHNR, que a variância residual fosse variável no nível ambiental médio (solução de grupo de contemporâneos).

A variância genética aditiva num ambiente $X\left(\sigma_{A}^{2} \mid X\right)$ foi obtida pela equação:

$$
\sigma_{A}^{2} \mid X=\sigma_{a}^{2}+X^{2} \sigma_{b}^{2}+2 X \sigma_{a b}
$$

Os efeitos residuais para o MHNR são presumidos com distribuição normal de média nula e com variâncias dadas por $\mathbf{R}=\mathbf{I} \sigma_{e}^{2}$, de modo que $\sigma_{e}^{2}$ é a variância residual e $\mathbf{I}$ representa uma matriz de identidade. Para o MHNRH, no entanto, os efeitos residuais assumem distribuição normal, ainda com média nula e independência entre resíduos de animais diferentes, mas com variância residual heterogênea, dada por $\mathbf{R}=\operatorname{diag}\left(\sigma_{e_{j}}^{2}\right)$, uma matriz diagonal com variâncias especificas para cada nível ambiental, onde $\sigma_{e_{j}}^{2}=\sigma_{e}^{2} \times \eta^{\hat{X}_{(g c) j}}$ e $\eta$ é o parâmetro de heterogeneidade de variância no gradiente ambiental, seguindo o modelo estrutural proposto por Cardoso et al. (2005b).

Portanto, a herdabilidade é estimada pelo quociente da variância genética pela variância fenotípica (genética + ambiental), como segue:

$$
h_{A}^{2} \mid X=\frac{\sigma_{A}^{2} \mid X}{\sigma_{A}^{2}\left|X+\sigma_{e}^{2}\right| X},
$$

na qual: $\sigma_{e}^{2} \mid X$ é a variância residual no ambiente $X$ dada por $\sigma_{e}^{2} \times \hat{\eta}^{X}$ no MHNRH e simplesmente por_ $\sigma_{e}^{2}$ em MA e MHNR.

As estimativas dos parâmetros foram obtidas usando-se uma abordagem bayesiana, por meio de métodos Monte Carlo via cadeias de Markov (MCMC) (Sorensen \& Gianola, 2002) com cadeias de 100.000 ciclos após período de descarte de 10.000 ciclos, de modo que as amostras foram retiradas a cada 10 ciclos. Médias, desvios-padrão e percentis $(0,025$ e 0,975$)$ a posteriori dos parâmetros foram obtidos das suas densidades posteriores marginais obtidas pelo Procedimento KDE do SAS (SAS, 2002).

$\mathrm{O}$ ajuste dos modelos (MA, MHNR e MHNRH) aos dados foi avaliado por meio de três critérios bayesianos:

- critério de informação da deviance (DIC) (Spiegelhalter et al., 2002): é um meio de comparação de modelos que segue a proposição de Dempster (1974), o qual sugere que comparações entre modelos sejam baseadas na distribuição a posteriori da deviance de cada modelo. O DIC é composto por uma medida de ajuste global, média a posteriori da deviance, e uma penalização por complexidade do modelo (número efetivo de parâmetros, $p_{D}$ ), que é calculada pela diferença. A diferença entre a média a posteriori da deviance e a deviance é baseada na média a posteriori dos parâmetros do modelo.

A deviance do modelo $i$ pode ser definida como:

$$
D(\boldsymbol{\theta})_{i}=-2 \log p\left(\mathbf{y} \mid \boldsymbol{\theta}, M_{i}\right)
$$

Um estimador de Monte Carlo é obtido por:

$$
\bar{D}(\boldsymbol{\theta})_{i}=\frac{1}{m} \sum_{j=1}^{m}-2 \log p\left(\mathbf{y} \mid \boldsymbol{\theta}^{(j)}, M_{i}\right)
$$

A complexidade do modelo $i$ como número efetivo de parâmetros é determinada por:

$$
p_{D i}=\bar{D}(\boldsymbol{\theta})_{i}-D(\overline{\boldsymbol{\theta}})_{i}
$$

em que $D(\overline{\boldsymbol{\theta}})_{i}=-2 \log p\left(\mathbf{y} \mid \overline{\boldsymbol{\theta}}, M_{i}\right)$ e $\overline{\boldsymbol{\theta}}$ é a média a posteriori dos parâmetros. Finalmente, o DIC é calculado por:

$$
D I C_{i}=\bar{D}(\boldsymbol{\theta})_{i}+p_{D i}
$$

Obtém-se $\log p\left(\mathbf{y} \mid \boldsymbol{\theta}^{(j)}, M_{i}\right)=\sum_{k=1}^{n} \log p\left(y_{k} \mid \boldsymbol{\theta}^{(j)}, M_{i}\right)$ e, ao final de $m$ ciclos, avalia-se $\overline{\boldsymbol{\theta}}=\frac{1}{m} \sum_{j=1}^{m} \boldsymbol{\theta}^{(j)}$ para obter $D(\overline{\boldsymbol{\theta}})_{i}=\sum_{k=1}^{n}-2 \log p\left(y_{k} \mid \overline{\boldsymbol{\theta}}, M_{i}\right)$, em que $D I C_{i}=2 \bar{D}(\boldsymbol{\theta})_{i}-D(\overline{\boldsymbol{\theta}})_{i}$. 
Menores valores de DIC indicam melhor ajuste do modelo, indicando um modelo mais próximo do modelo ideal.

- Deviance baseada nos fatores de Bayes (BF): o fator de Bayes é uma medida global de ajuste dada pela razão entre as distribuições marginais dos dados $p\left(\mathbf{y} \mid M_{i}\right)$ sob dois modelos diferentes $\left(M_{i}, i=1,2\right)$, de modo que:

$$
B F=\frac{p\left(\mathbf{y} \mid M_{1}\right)}{p\left(\mathbf{y} \mid M_{2}\right)}
$$

Pode ser demonstrado que $p\left(\mathbf{y} \mid M_{i}\right)=E^{-1}\left[p^{-1}\left(\mathbf{y} \mid \boldsymbol{\theta}, M_{i}\right)\right]$ e um estimador de Monte Carlo foi proposto por Newton \& Raftery (1994):

$$
\hat{p}\left(\mathbf{y} \mid M_{i}\right)=\frac{1}{\frac{1}{m} \sum_{j=1}^{m} p^{-1}\left(\mathbf{y} \mid \boldsymbol{\theta}^{(j)}, M_{i}\right)}
$$

no qual $m$ é número do ciclos de Gibbs e $\boldsymbol{\theta}^{(j)}$, amostra da distribuição a posteriori dos parâmetros no ciclo $j$. Para evitar erros de precisão ao calcular $B F$, calculou-se $f_{i}=\frac{1}{m} \sum_{j=1}^{m} \exp \left(-\log p\left(\mathbf{y} \mid \boldsymbol{\theta}^{(j)}, M_{i}\right)-c\right) \exp c$, em que $c$ é o maior valor de $-\log p\left(\mathbf{y} \mid \boldsymbol{\theta}^{(j)}, M_{i}\right)$ e tomando-se o logaritmo $\log f_{i}=\log \left[\frac{1}{m} \sum_{j=1}^{m} \exp \left(-\log p\left(\mathbf{y} \mid \boldsymbol{\theta}^{(j)}, M_{i}\right)-c\right)\right]+c$ e finalmente: $B F=\exp \left(-\log f_{1}+\log f_{2}\right)$.

A cada ciclo de Gibbs, salvou-se o $\log p\left(\mathbf{y} \mid \boldsymbol{\theta}^{(j)}, M_{i}\right)=\sum_{k=1}^{n} \log p\left(y_{k} \mid \boldsymbol{\theta}^{(j)}, M_{i}\right)$ e, no final dos $m$ ciclos, o $B F$ pôde ser obtido como descrito acima, de modo que valores maiores que 1 suportam $M_{1}$, enquanto valores menores suportam $M_{2}$.

A deviance baseada no estimador do $B F$ para o modelo $i$ é igual a $-2 \log f_{i}$ e menores valores dessa deviance indicam melhor ajuste.

- Deviance baseada na Ordenada Preditiva Condicional - Conditional Predictive Ordinate (CPO): os CPO são densidades de validação cruzada $p\left(y_{k} \mid \mathbf{y}_{(k)}\right)$, que sugerem quais valores de $y_{k}$ são prováveis quando o modelo é ajustado a $\mathbf{y}_{(k)}$, o conjunto de todas as observações exceto $y_{k}$. O CPO proporciona uma medida de ajuste para cada observação individualmente e comparações entre modelos são feitas por razões de $C P O$ :

$$
C_{k}=\frac{p\left(y_{k} \mid \mathbf{y}_{(k)}, M_{1}\right)}{p\left(y_{k} \mid \mathbf{y}_{(k)}, M_{2}\right)}
$$

Adicionalmente, uma medida global de ajuste é determinada pelo pseudofator de Bayes:

$$
P B F=\prod_{k=1}^{n} C_{k}=\prod_{k=1}^{n} \frac{p\left(y_{k} \mid \mathbf{y}_{(k)}, M_{1}\right)}{p\left(y_{k} \mid \mathbf{y}_{(k)}, M_{2}\right)}
$$

Desde que os $y_{k}$ sejam condicionalmente independentes, dado $\boldsymbol{\theta}$, o CPO pode ser estimado por Monte Carlo da seguinte forma:

$$
C P O_{k}=p\left(y_{k} \mid \mathbf{y}_{(k)}, M_{i}\right)=\frac{1}{\frac{1}{m} \sum_{j=1}^{m} p^{-1}\left(y_{k} \mid \boldsymbol{\theta}^{(j)}, M_{i}\right)}
$$

O programa INTERGEN avalia a cada ciclo $p\left(y_{k} \mid \boldsymbol{\theta}^{(j)}, M_{i}\right)$, mantendo uma soma corrente desses valores para no final calcular a média harmônica acima.

Finalmente o estimador da deviance do modelo $i$ baseada em CPO é obtido por $-2 \sum_{k=1}^{n} \log \hat{p}\left(y_{k} \mid \mathbf{y}_{(k)}, M_{i}\right)$, para o qual menores valores indicam melhor ajuste.

A análise de convergência global das cadeias para os diferentes modelos foi realizada por meio do diagnóstico de Geweke (1992), baseado em um teste $Z$ de igualdade de médias do logaritmo da distribuição condicional dos dados denotados por $l_{i}^{(j)}=\log p\left(\mathbf{y} \mid \boldsymbol{\theta}^{(j)}, M_{i}\right)$ das primeiras amostras (10\% iniciais) e da última parte da cadeia de Markov (últimas 50\%), similar ao proposto por Brooks \& Roberts (1998), utilizando-se a seguinte estatística:

$$
Z_{i}=\frac{\left(\bar{l}_{i}^{A}-\bar{l}_{i}^{B}\right)}{\sqrt{\frac{1}{n_{A}} \hat{S}_{i}^{A}(0)+\frac{1}{n_{B}} \hat{S}_{i}^{B}(0)}},
$$

em que: $\bar{l}_{i}^{A}=\frac{1}{n_{A}} \sum_{j=1}^{n_{A}} l_{i}^{(j)}, \bar{l}_{i}^{B}=\frac{1}{n_{B}} \sum_{j=n^{*}}^{m} l_{i}^{(j)}, n_{A}=10.000$, $n_{B}=50.000, n^{*}=50.001$, e com $\hat{S}_{i}^{A}(0)$ e $\hat{S}_{i}^{B}(0)$, de modo que as respectivas estimativas da densidade espectral na frequência zero foram obtida pelo Procedimento SPECTRA do SAS (SAS, 2002) para os primeiros $n_{A}$ e os últimos $n_{B}$ ciclos da cadeia MCMC de comprimento $m$. Valores absolutos extremos do escore $Z_{i}$, para um teste de duas caudas, indicam rejeição do teste de convergência.

Além disso, critérios baseado em Geyer (1992) e Sorensen et al. (1995) foram utilizados para calcular, respectivamente, a variância de Monte Carlo e o número efetivo de amostras. Considerando a seqüência de amostras da cadeia $\theta_{1}, \theta_{2}, \ldots, \theta_{m}$, em que $m$ é o número de ciclos e $q_{i}$ é a amostra no ciclo $i$, a autocovariância temporal da seqüência é estimada por:

$$
\hat{\gamma}_{m}(t)=\frac{1}{m} \sum_{i=1}^{i=m-t}\left(\theta_{i}-\bar{\theta}\right)\left(\theta_{i+t}-\bar{\theta}\right)
$$

em que $\bar{\theta}=\frac{1}{m} \sum_{i=1}^{i=m} \theta_{i}$ é a média amostral para a cadeia e $t$ é o atraso. 
Um estimador da variância da média amostral proposto por Geyer (1992), baseado em séries temporais, é o estimador da sequência positiva inicial. Dada a função $\hat{\Gamma}_{m}(t)=\hat{\gamma}_{m}(2 t)+\hat{\gamma}_{m}(2 t+1), t=0,1, \ldots$, o estimador é definido como:

$$
m(\operatorname{var}(\bar{\theta}))=\hat{\gamma}_{m}(0)+2 \sum_{i=1}^{2 t+1} \hat{\gamma}_{m}(i)=-\hat{\gamma}_{m}(0)+2 \sum_{i=0}^{t} \hat{\Gamma}_{m}(i)
$$

em que $t$ é o maior número inteiro que satisfaça $\hat{\Gamma}_{m}(i)>\mathbf{0}, \quad i=0,1, \ldots, t$. O número efetivo de amostras independentes (Sorensen et al., 1995) é determinado por:

$$
\hat{\psi}_{m}=\frac{\hat{\gamma}_{m}(0)}{\operatorname{var}(\bar{\theta})} .
$$

\section{Resultados e Discussão}

Os resultados de desempenho foram obtidos pelas análises descritivas dos dados nas etapas de formatação e consistência desses dados (Tabela 1). A conectabilidade dos grupos de contemporâneos resultou no arquipélago 1 (grupo de contemporâneo com maior número de laços genéticos e todos grupos de contemporâneos a ele conectados) com 14.973 animais em 852 GC, 41 animais em 6 GC no arquipélago 2 e 496 animais e 52 GC desconectados.

Os testes globais para avaliar a convergência das cadeias MCMC para o modelo animal, para o modelo hierárquico normas de reação e para o modelo hierárquico normas de reação heterocedástico (Tabela 2) apontam para convergência à distribuição posterior estacionária.

Tabela 1 - Médias, desvios-padrão e amplitudes observadas para características de desempenho de bovinos Devon

\begin{tabular}{lcccc}
\hline Característica & Média $(\mathrm{kg})$ & $\begin{array}{c}\text { Desvio- } \\
\text { padrão }\end{array}$ & Mínimo & Máximo \\
\hline Peso à desmama & 162,70 & 37,10 & 80 & 336 \\
$\begin{array}{l}\text { Peso ao sobreano } \\
\text { Ganho pós-desmama }\end{array}$ & 104,34 & 63,21 & 140 & 630 \\
$\begin{array}{l}\text { Ganho diário } \\
\text { pós-desmama }\end{array}$ & 0,3312 & 44,15 & 17 & 360 \\
$\begin{array}{l}\text { Ganho pós-desmama } \\
\text { padronizado para 345 dias }\end{array}$ & 114,26 & 50,29 & 34,83 & 482,26 \\
\hline
\end{tabular}

Pelos resultados obtidos nos critérios utilizados para comparação dos modelos, como a deviance baseada nos fatores de Bayes (FB), o critério de informação da deviance (DIC) e a deviance baseada na ordenada preditiva condicional (CPO), o MHNR foi o que melhor se ajustou aos dados e foi o melhor modelo em todos esses critérios (Tabela 2).

Pelo programa INTERGEN, obtiveram-se resultados dos componentes de variância e parâmetros genéticos para GPD345 considerando os três modelos propostos. O número efetivo de amostras para as distribuições posteriores marginais (Figura 1) e para o parâmetro de heterogeneidade de variância residual (Figura 2) variou entre 128 e 7619, com média de 598 amostras.

No modelo hierárquico normas de reação heterocedástico, a média \pm desvio-padrão posterior (com intervalo de $95 \%$ de probabilidade posterior entre parênteses) da variância genética obtida para o intercepto do animal ou nível da norma de reação (Figura 1B) foi de 177,86 $\pm 16,60 \mathrm{~kg}^{2}$ $(144,85 ; 211,38)$ e para a inclinação da norma de reação (Figura 1C), de $0,076 \pm 0,006 \mathrm{~kg}^{2}(0,066 ; 0,087)$. A variância residual heterogênea também cresceu com o gradiente ambiental e foi obtida por $\sigma_{e}^{2} \mid X=401,96 \times 1,00986^{X}$, em que $401,96 \pm 11,91 \mathrm{~kg}^{2}(378,65 ; 425,82)$ é o valor de $\sigma_{e}^{2}$ (Figura 1E) e 1,00986 $\pm 0,00057(1,0087 ; 1,0110)$ a média posterior do parâmetro de heterogeneidade $\eta$ (Figura 2). A correlação entre nível e inclinação da norma neste modelo MHNRH (Figura 1D) foi de $0,72 \pm 0,023(0.678 ; 0,768)$.

As variâncias genéticas obtidas no modelo hierárquico normas de reação foram de $340,38 \pm 17,11 \mathrm{~kg}^{2}$ $(309,09 ; 375,27)$ e $0,119 \pm 0,006 \mathrm{~kg}^{2}(0,107 ; 0,130)$, respectivamente, para o nível (Figura 1B) e a inclinação da norma de reação (Figura 1C); e a correlação genética entre nível e inclinação foi de $0,83 \pm 0,01(0,817 ; 0,858)$, superior à encontrada por meio do MHNRH, mas ambas indicaram que os animais de maior valor genético no ambiente médio $(X=0)$ foram também os que mais responderam à melhora no ambiente.

Finalmente, para o modelo animal, a variância genética do efeito de animal foi de $73,47 \pm 9,89 \mathrm{~kg}^{2}(54,20 ; 93,64)$. Essa variância pode ser comparada à variância do nível da

Tabela 2 - Teste de convergência de Geweke (Z), critério de Informação da deviance (DIC), deviance baseada na ordenada preditiva condicional (CPO) e deviance baseada nos fatores de Bayes (FB) para comparação entre o modelo animal convencional (MA),

\begin{tabular}{|c|c|c|c|c|}
\hline Modelo & $\mathrm{DIC}$ & $\mathrm{CPO}$ & FB & Z (p-valor) \\
\hline Modelo animal (MA) & $137.490,9$ & 137.842 & $136.013,4$ & $0,89 \quad(0,3752)$ \\
\hline Modelo hierárquico normas de reação (MHNR) & $133.120,8$ & $135.879,6$ & $127.861,1$ & $1,06(0,2911)$ \\
\hline Modelo hierárquico normas de reação heterocedástico (MHNRH) & $135.650,2$ & 136.500 & $132.339,8$ & $1,22(0,2218)$ \\
\hline
\end{tabular}
e diferentes modelos 
A

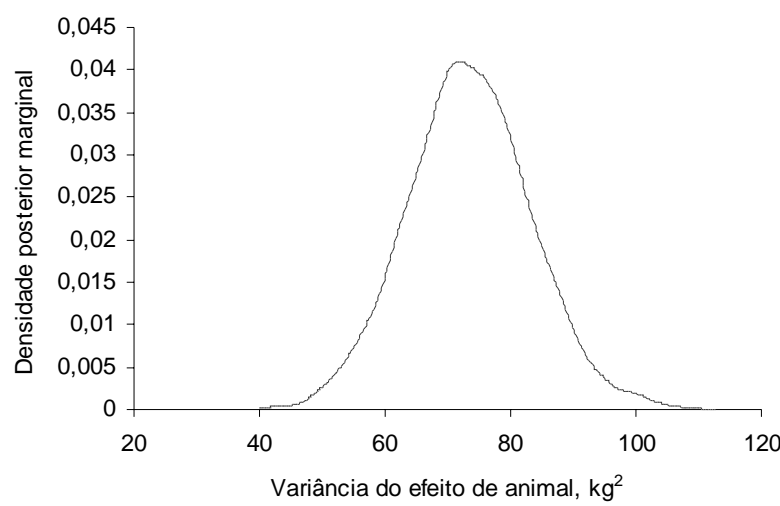

C

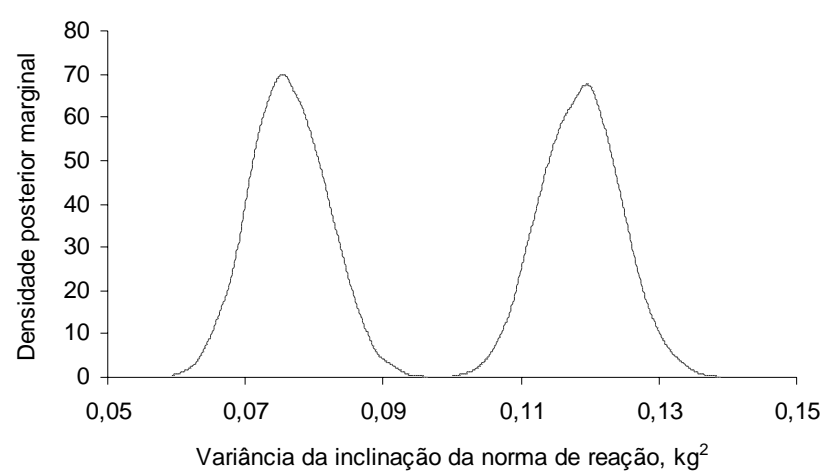

E

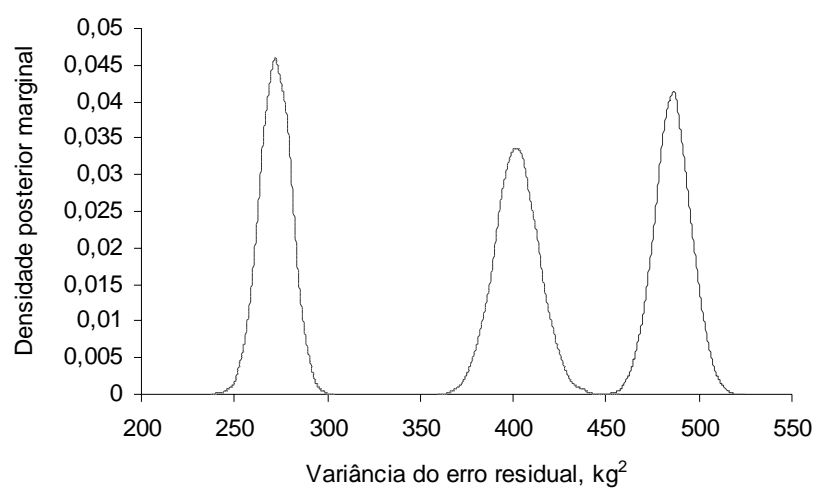

B

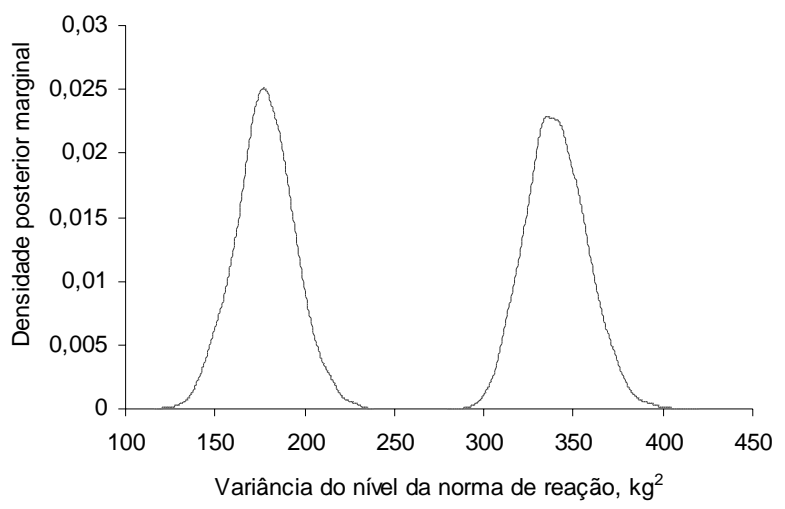

D

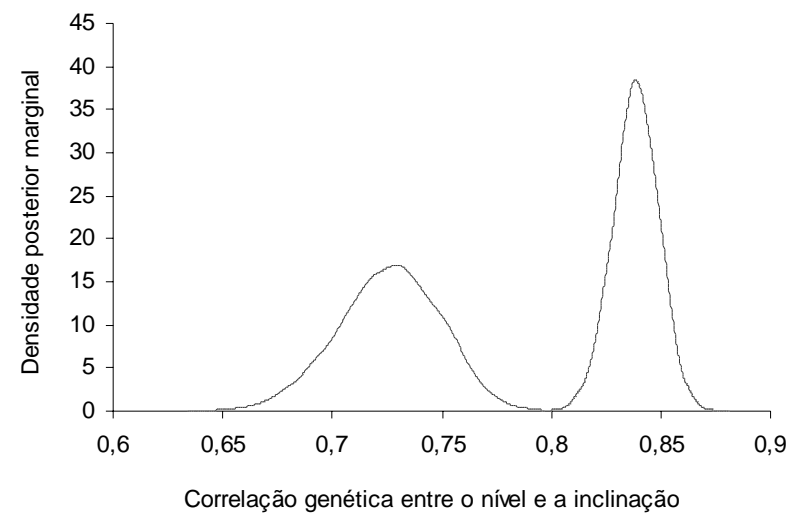

F

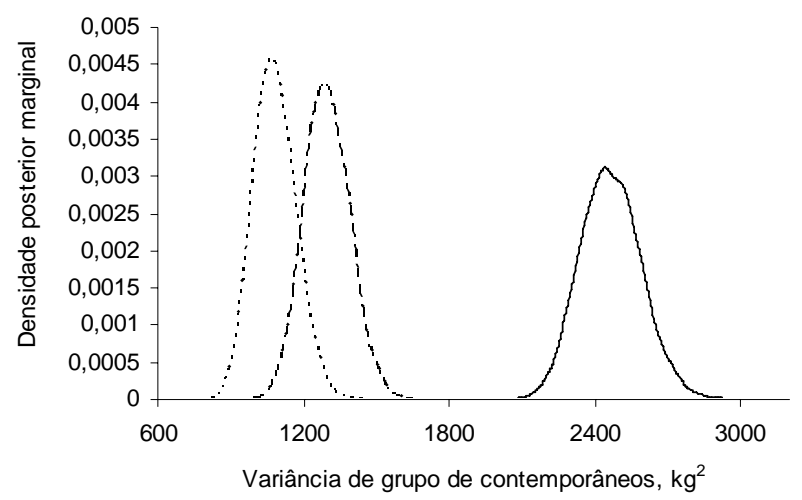

Figura 1 - Distribuição posterior marginal das variâncias dos efeitos de animal (A), de nível da norma de reação (B), de inclinação da norma de reação $(\mathrm{C})$, dos erros residuais (E) e de grupo de contemporâneos (F), e da correlação genética entre o nível e a inclinação da norma de reação (D) para modelo animal (MA), modelo hierárquico de normas de reação (MHNR) e modelo hierárquico normas de reação heterocedástico $(\mathrm{MHNRH})$.

norma de reação em um modelo que todas as inclinações dos animais são fixadas em zero onde se observa que no presente estudo o seu valor foi inferior aos obtidos pelo MHNR e MHNRH.

O gradiente ambiental obtido pelas soluções dos efeitos de grupo de contemporâneos no modelo animal convencional variou entre $-73,6$ e $243,1 \mathrm{~kg}$ e $80 \%$ dessas soluções foram entre -51,3 (percentil 0,10) e 71,4 (percentil 0,90). As variâncias genéticas aditivas para a característica avaliada GPD345 variaram conforme esse gradiente ambiental, aumentando à medida que melhorou o ambiente, o que comprova efeito em escala de interação genótipo-ambiente (Figura 3), tanto no modelo hierárquico normas de reação (MHNR) como para o modelo hierárquico normas de reação heterocedástico (MHNRH).

A variação observada nas variâncias genéticas aditivas no ambiente $X$ está de acordo com os resultados encontrados por Cardoso et al. (2005a), Kolmodin et al. (2002) e Su et al. (2006) e Cardoso et al. (2007), que observaram que essas variâncias aumentaram conforme o gradiente 


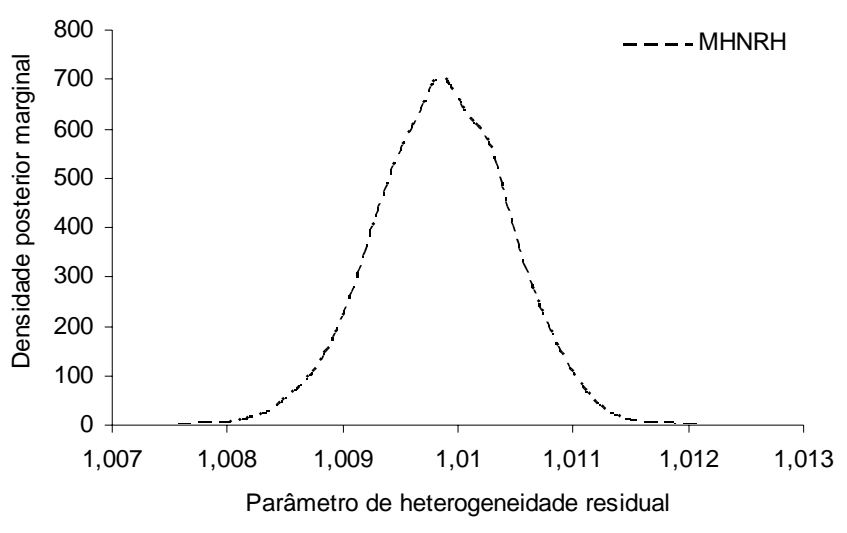

Figura 2 - Distribuição posterior marginal do parâmetro de heterogeneidade da variância residual no modelo hierárquico normas de reação heterocedástico (MHNRH).

ambiental se tornou favorável, acentuando as diferenças entre os indivíduos mais responsivos.

A herdabilidade foi de $0,13 \pm 0,017$ para o modelo animal (MA). Esse valor está abaixo do encontrado por Cardoso et. al. (2004) e Cardoso et al. (2005a) para o ganho

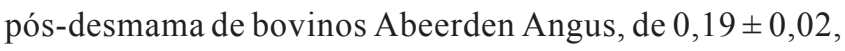
e Cardoso et al. (2007), de 0,18 $\pm 0,01$ num conjunto de dados diferentes da mesma população Abeerden Angus.

Da mesma forma que as variâncias, nos modelos MHNR e MHNRH, as herdabilidades aumentaram conforme a melhora do gradiente ambiental (Figura 4), o que comprova modificação nos parâmetros genéticos da população de acordo com o ambiente ao qual estão expostos, caracterizando a presença de interação genótipo-ambiente. Assim, considerou-se como nível ambiental baixo o valor do primeiro quartil da distribuição das soluções de grupo de contemporâneos, ou seja, 25\% dos grupo de contemporâneos com piores valores ambientais $(X=-36,5 \mathrm{~kg})$, como nível médio no qual $\mathrm{X}=0 \mathrm{~kg}$, ou seja, a média de efeito ambiental e como nível ambiental alto, $25 \%$ dos grupo de contemporâneos com melhores valores ambientais (terceiro quartil) $\operatorname{com} X=21,8 \mathrm{~kg}$.

Para o modelo hierárquico normas de reação (MHNR), os valores das herdabilidades foram bastante superiores à herdabilidade no modelo animal (MA): para o nível baixo, foram de $0,29 \pm 0,020$; para o nível médio, de $0,56 \pm 0,018$; e para o nível alto, de $0,70 \pm 0,015$. Essas herdabilidades são superiores às encontradas por Cardoso et al. (2007), de $0,18,0,29 \pm 0,02$ e 0,45 , respectivamente, para os níveis baixo, médio e alto, utilizando um modelo hierárquico normas de reação para avaliar o ganho pós-desmama em bovinos Abeerden Angus.
A

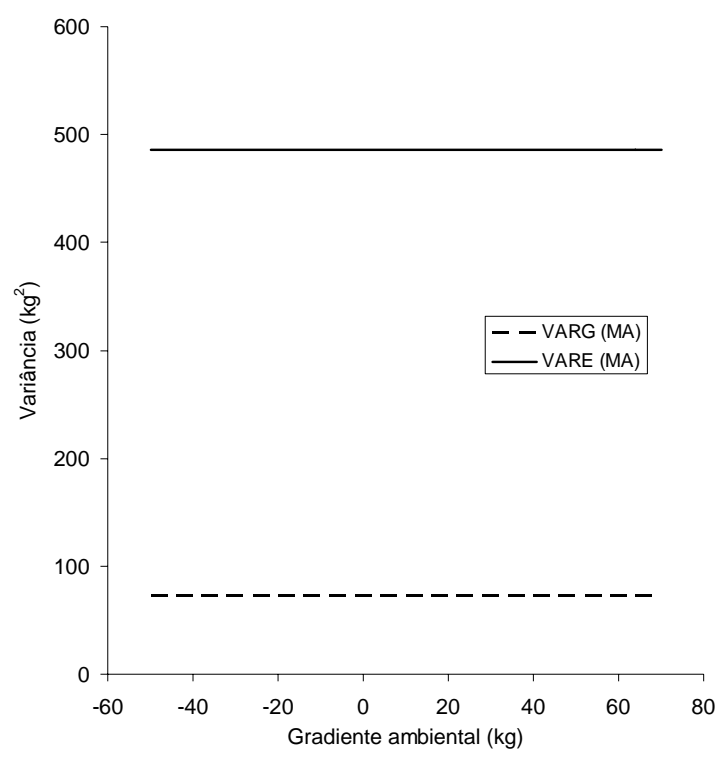

B

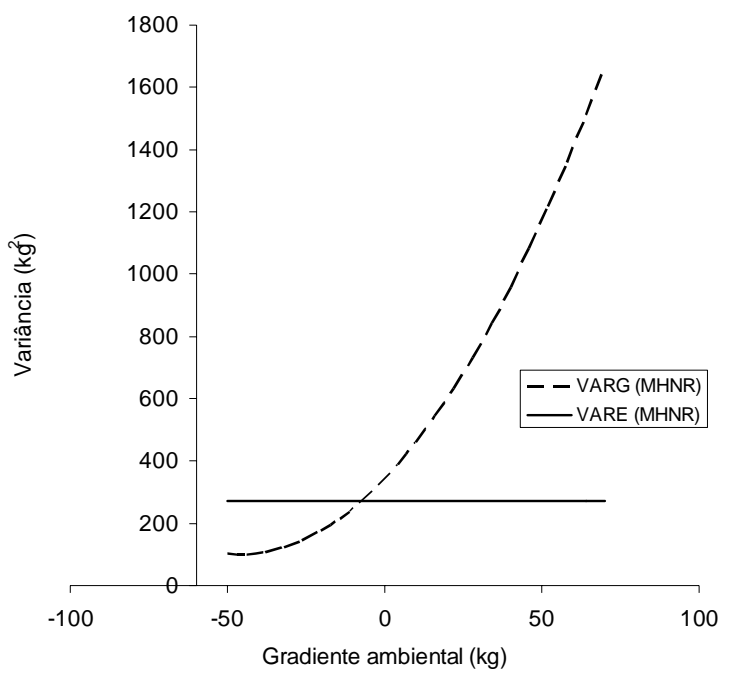

C

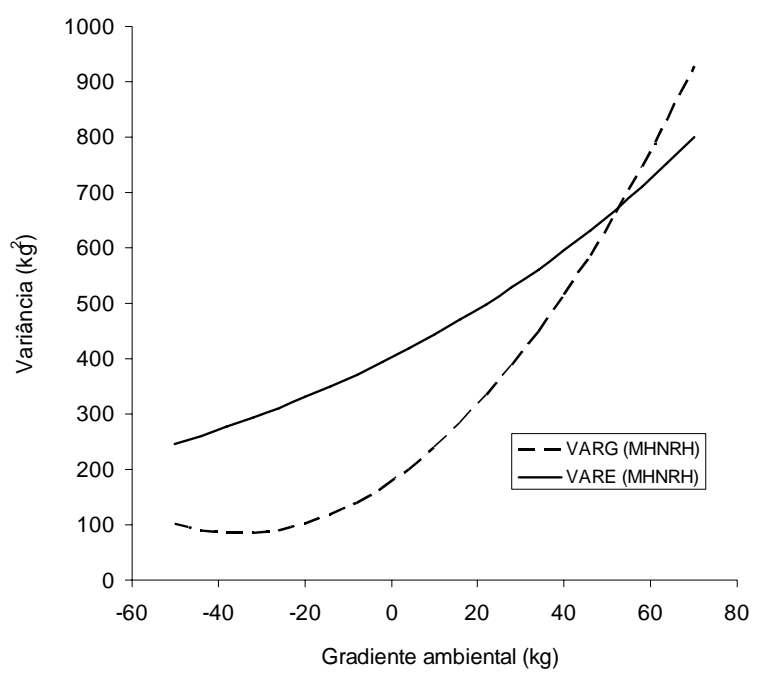

Figura 3 - Variâncias genéticas (VARG) e residuais (VARE) conforme o gradiente ambiental obtidas pelo modelo animal (MA, Painel A), modelo hierárquico normas de reação (MHNR, Painel B) e no modelo hierárquico normas de reação heterocedástico (MHNRH, Painel C). 


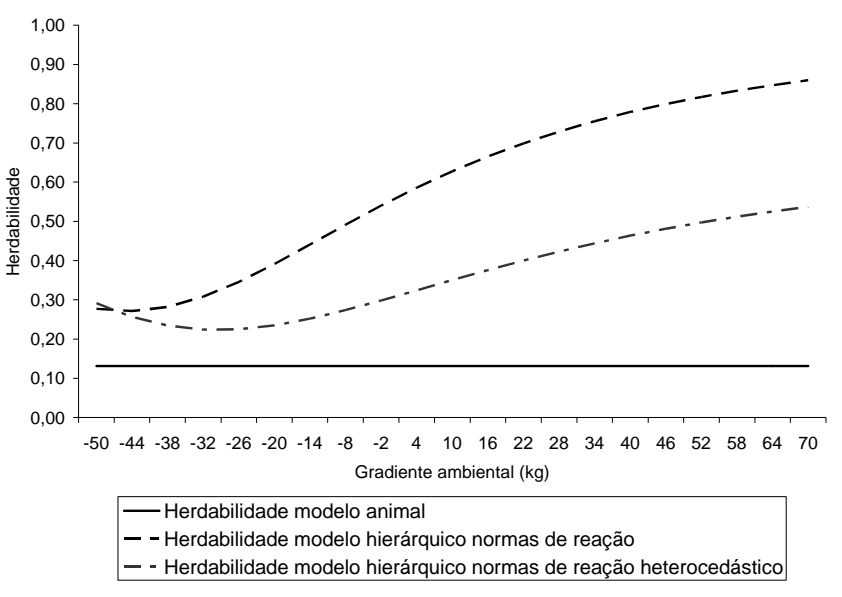

Figura 4 - Herdabilidades da característica GDP345 nos modelos propostos pelo gradiente ambiental.

No modelo hierárquico normas de reação heterocedástico (MHNRH), os valores das herdabilidades foram intermediários, de $0,23 \pm 0,018$ no nível baixo; $0,31 \pm 0,025$ no nível médio e $0,40 \pm 0,028$ no nível alto, em comparação ao obtido pelo modelo animal e pelo MHNR.

A atribuição da variação fenotípica a fatores genéticos foi maior que aos fatores ambientais pelos modelos hierárquicos, o que aponta melhor resposta à seleção em relação ao modelo animal (Figura 4). Entre os modelos hierárquicos, observa-se relação de aumento da herdabilidade muito similar nos valores positivos do gradiente ambiental, pois, mesmo que no MHNR a variância residual fosse constante, o crescimento da relação entre variância genética e a fenotípica foi similar ao do MHNRH, que tem variância residual heterocedástica, conforme observado pela diferença de herdabilidade em favor do MHNR praticamente constantes nessa faixa do gradiente ambiental.

A correlação genética nos valores médios e altos do gradiente ambiental é altamente positiva, acima de 0,80 (Figura 5), entretanto, cai rapidamente à medida que 0 ambiente se torna desfavorável, apresentado valores negativos entre os extremos do gradiente ambiental. Esses resultados indicam que a genética necessária para produção nos ambientes muito adversos é diferente daquela que produz melhor nos ambientes razoáveis e bons.

Nos modelos MHNR e MHNRH, o comportamento foi o mesmo, com variância genética aditiva crescente à medida que o gradiente ambiental se torna favorável. Isso indica que os animais de maior valor genético médio para GPD345 foram os que mais responderam à melhora de ambiente, o que está de acordo com observações de Cardoso et. al. (2005a) para o ganho pós-desmama em bovinos Abeerden Angus e de Kolmodin et al. (2002) para produção de proteína em bovinos de leite.
Correlação genética no MHNR

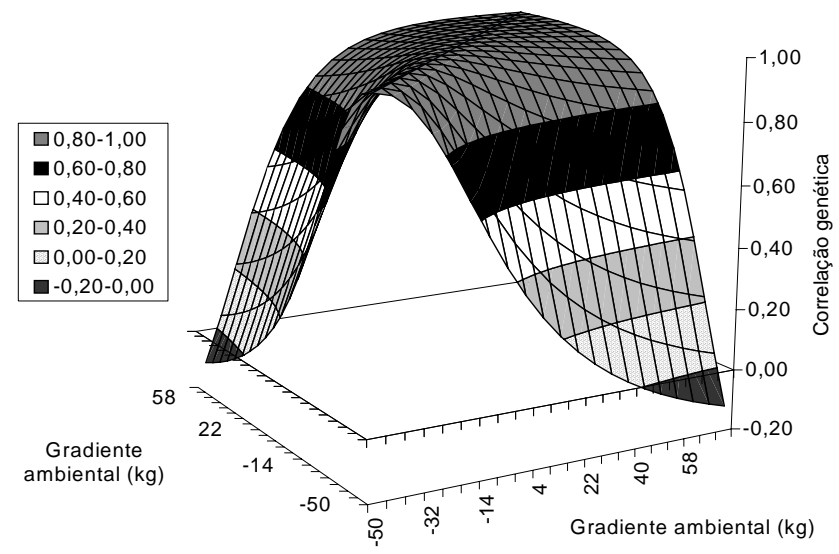

Figura 5 - Correlação genética no modelo hierárquico normas de reação (MHNR).

Os resultados também estão de acordo com os obtidos por Calus \& Veerkamp (2003) para características de produção de leite, produção de proteína e gordura no leite, onde as herdabilidades aumentaram conforme o gradiente ambiental, da mesma forma demonstrando efeito em escala. As variâncias genéticas tiveram considerável efeito em escala, deixando explícita grande diferença de sensibilidade ambiental entre os indivíduos.

Pollott \& Greeff (2004), avaliando características de produção (peso corporal, peso de velo) e de resistência a parasitas (contagem de ovos nas fezes) em ovinos Merino Australiano por modelo polinomial de regressão aleatória, também demonstraram efeito de interação $G^{*} A$. As herdabilidades variaram conforme o ambiente para as características de peso corporal e a contagem de ovos nas fezes, evidenciando claramente a interação $G^{*} A$.

Os resultados para as soluções ambientais dos grupos de contemporâneos variaram, no modelo animal padrão de -73,57 a 243,05; no MHNR de -61,28 a 99,81; e no MHNRH de $-65,01$ a 104,40. Isso demonstra que a maior variação foi observada no modelo animal, seguido do MHNRH e finalmente do MHNR (Figura 1F), e, nos dois últimos, foi praticamente a mesma, confirmando que não há necessidade de utilização do MHNRH, uma vez que as soluções ambientais no MHNR, que melhor ajustou os dados, foram de mesma magnitude.

As soluções para os valores genéticos variaram significativamente. Tanto no MHNR quanto no MHNRH, houve variação bastante superior dos valores genéticos no nível alto do gradiente ambiental em comparação ao nível baixo. No MHNR no nível baixo, variou de -18,69 a 37,59, uma diferença de 56,28 entre os extremos. No nível alto, entretanto, variou de $-69,86$ a 94,04, diferença de 163,90 . No MHNRH, a variação no nível baixo ficou entre -14,56 e 24,84, 
diferença de 39,40, enquanto, no nível alto a variação ficou entre $-55,85$ e 67,17 , diferença de 123,02 .

Os resultados deste estudo permitem concluir, de acordo com diversos autores (Kolmodin et al., 2002; Calus \& Veerkamp, 2003; Calus et al., 2004; Pollott \& Greeff, 2004; Cardoso et al., 2005a; Su et al., 2006), que há interação genótipo-ambiente na população Devon estudada essencialmente em virtude do efeito de escala, pois, quando consideradas as normas de reação, os valores genéticos apresentaram maior variação nos valores positivos do gradiente ambiental em comparação aos níveis ambientais mais baixos. Ou seja, à medida que o ambiente se torna mais favorável, aumenta a diferença entre os valores genéticos dos indivíduos, uma vez que esses indivíduos expressam melhor seu potencial.

\section{Conclusões}

Existe interação genótipo-ambiente na população de bovinos Devon no Rio Grande do Sul. Na população avaliada, o modelo que melhor se ajustou aos dados foi o de normas de reação com variância residual homogênea. A maior herdabilidade observada para os modelos de normas de reação indica que o uso desses modelos em programas de avaliação genética pode aumentar o progresso genético na população estudada. No entanto, estudos adicionais são necessários para verificar a viabilidade da implementação prática das normas de reação no processo de estimativa de valores genéticos para ambientes específicos e as consequências da seleção considerando a sensibilidade ambiental a médio e longo prazo.

\section{Agradecimentos}

À Associação Nacional de Criadores Herd Book Collares e ao técnico Leonardo Talavera Campos, pela cessão dos dados utilizados na pesquisa. À CAPES, em especial, pela concessão da bolsa de estudos.

\section{Literatura Citada}

BOLDMAN, K.G.; FREEMAN, A.E. Adjustment for heterogeneity of variances by herd production level in dairy cow and sire evaluation. Journal of Dairy Science, v.73, p.503-512, 1990.

BROOKS, S.P.; ROBERTS, G.O. Convergence assessment techniques for Markov chain Monte Carlo. Statistics and Computing, v.8, n.4, p.319-335, 1998.

CALUS, M.P.L.; VEERKAMP, R.F. Estimation of environmental sensitivity of genetic merit for milk production traits using a random regression model. Journal of Dairy Science, v.86, p.3756-3764, 2003 .

CALUS, M.P.L.; BIJMA, P.; VEERKAMP, P.R.F. Effects of data structure on the estimation of covariance functions to describe genotype by environment interactions a reaction norm model. Genetic Select Evolution, v.36, p.489-507, 2004.

CARDOSO, F.F.; CARDELlinO, R.A.; CAMPOS, L.T. Componentes de (co)variância e parâmetros genéticos de caracteres pós-desmama em bovinos da Raça Angus. Revista Brasileira de Zootecnia, v.33, n.2, p.313-319, 2004.

CARDOSO, F.F.; CAMPOS, L.T.; CARDELLINO, R.A. Caracterização de interação genótipo-ambiente no ganho pós-desmama de bovinos Angus via normas de reação "1". In: REUNIÃO ANUAL DA SOCIEDADE BRASILEIRA DE ZOOTECNIA 42., 2005 , Goiânia. Anais ... Goiânia: Sociedade Brasileira de Zootecnia, 2005a. (CD-ROM).

CARDOSO, F.F.; ROSA, G.J.M.; TEMPELMAN, ER.J. Multiplebreed genetic inference using heavy-tailed structural models for heterogeneous residual variances. Journal of Animal Science, v.83, n.8, p.1766-1779, 2005b.

CARDOSO, F.F.; OLIVEIRA, M.M.; CAMPOS, L.T. Modelos hierárquicos bayesianos para estudo da interação genótipoambiente via normas de reação aplicados ao ganho pós desmama de bovinos Angus. In: REUNIÃO ANUAL DA SOCIEDADE BRASILEIRA DE ZOOTECNIA 44., 2007, Jaboticabal. Anais... Jaboticabal: Sociedade Brasileira de Zootecnia, 2007. p.608.

CARDOSO, F.F. Manual de utilização do programa INTERGEN - Versão $1.0 \mathrm{em}$ estudos de genética quantitativa animal. Bagé: Embrapa Pecuária Sul, 2008. p.74.

CAVALHEIRO, R.; FRIES, L.A.; SCHENKEL, F. S. et al. Efeitos da heterogeneidade de variância residual entre grupo de contemporâneos na avaliação genética de bovinos de corte Revista Brasileira de Zootecnia, v.31, n.4, p.1680-1688, 2002.

DEMPSTER, A.P. The direct use of likelihood for significance testing. In: CONFERENCE ON FOUNDATIONAL QUESTIONS IN STATISTICAL INFERENCE, 1974. Proceedings... University of Aarhus/Department of Theoretical Statistic, 1974 p.335-352.

De JONG, G. Phenotypic plasticity as a product of selection in a variable environment. American Naturalist, v.145, n.4 p.493-512, 1995.

DONG, M.C.; MAO, I.L. Heterogeneity of (co)variance and heritability in different levels of intraherd milk production variance and of herd average. Journal of Dairy Science, v.73, p.843-851, 1990

FALCONER, D.S.; MACKAY, T.F.C. Introduction to quantitative genetics. Harlow: Longman Group Ltda, 1996. 464p.

GARRICK, D.J.; Van VLECK, L.D. Aspects of selection for performance in several environments with heterogeneous variances. Journal of Animal Science, v.65, p.409-421, 1987.

GEWEKE, J. Evaluating the accuracy of sampling-basedapproaches to calculating posterior moments. In: BERNARDO, J.M. BERGER, J.O.; DAWID, A.P. et al. (Eds.) Bayesian statistics. Oxford: Clarendon Press, 1992. v.4.

GEYER, C.J. Practical Markov chain Monte Carlo. Statistical Science, v.7, n.4, p.473-511, 1992.

KOLMODIN, R.; STRAMBERG, E.; MADSEN, P. et al. Genotype by environment interaction in Nordic dairy cattle studied using reaction norms. Acta Agriculture Scandinavia, Section A, Animal Science, v.52, p.11-24, 2002.

MERCADANTE, M.E.Z.; PACKER, I.U.; RAZOOK, A.G. et al Dias ao parto de fêmeas Nelore de um experimento de seleção para crescimento. II - Modelo de regressão aleatória. Revista Brasileira de Zootecnia, v.31, n.4, p.1726-1733, 2002.

MEYER, K. Scope for a random regression model in genetic evaluation of beef cattle for growth. Livestock Production Science, v. 86, p.69-83, 2003.

NEWTON, M.A.; RAFTERY, ADRIAN E. Approximate Bayesian Inference with the Weighted Likelihood Bootstrap. Journal of the Royal Statistical Society, v.56, n.1, p.33-48, 1994. 
POLLOTT, G.E.; GREEFF, J.C. Genotype $\mathrm{x}$ environment interactions and genetic parameters for fecal egg count and production traits of Merino sheep. Journal of Animal Science, v.82, p.2840-2851, 2004.

REVERTER, A.; TIER, B.; JOHNSTON, D.J. et al. Assessing the efficiency of multiplicative mixed model equations to account for heterogeneous variance across herds in carcass scan traits from beef cattle. Journal of Animal Science, v.75, p.1477-1485, 1997.

ROSO, V.M.; SHENKEL, F.S. AMC - A computer program to assess the degree of connectedness among contemporary groups. In: WORLD CONGRESS ON GENETICS APPLIED TO LIVESTOCK PRODUCTION, 8., 2006, Belo Horizonte. Proceedings... Belo Horizonte: 2006. p.27-26.

SORENSEN, D.A.; ANDERSEN, S.; GIANOLA, D. et al. Bayesianinference in threshold models using Gibbs sampling. Genetics Selection Evolution, v.27, n.3, p.229-249, 1995.
SORENSEN, D.A.; GIANOLA, D. Likelihood, Bayesian and MCMC methods in quantitative genetics. 1.ed. New York: Springer-Verlag New York Inc., 2002.

SPIEGELHALTER, D.J.; BEST, N.G.; CARLIN, B.P. et al. Bayesian measures of model complexity and fit. Journal of the Royal Statistical Society, Series B - Statistical Methodology, v.64, p.583-616, 2002.

STATISTICAL ANALYSIS SYSTEM - SAS. SAS 9.1.3 Help and Documentation. Cary: S AS Institute, 2002.

SU, G.; MADSEN, P.; LUND, M.S. et al. Bayesian analysis of the linear reaction norm model with unknown covariates. Journal of Animal Science, v.84, p.1651-1657, 2006.

TORRES, R.A. Efeito da heterogeneidade de variância na avaliação genética de bovinos da raça Holandesa no Brasil. 1998. 124f. Tese (Doutorado em Ciência Animal) Universidade Federal de Minas Gerais, Belo Horizonte, 1998. 